\title{
STUDY ON A LOOPED 2-STAGE THERMOACOUSTIC ENGINE
}

\author{
T. Jin ${ }^{1,2^{*}}$, R. Yang ${ }^{1}$, Y.L. Liu ${ }^{1}$, K. Tang ${ }^{1}$ and B.M. Chen ${ }^{3}$ \\ ${ }^{1}$ Institute of Refrigeration and Cryogenics, Zhejiang University, Hangzhou 310027, P.R.China. \\ ${ }^{2}$ State Key Laboratory of Clean Energy Utilization, Zhejiang University, Hangzhou 310027, China. \\ ${ }^{*}$ Corresponding author's e-mail: jintao@zju.edu.cn \\ ${ }^{3}$ Department of Energy and Chemical Engineering, Dongguan University of Technology, Dongguan 523808, \\ P.R.China.
}

Keywords: thermoacoustic prime mover, looped 2-stage configuration, onset temperature, low-grade heat source

Multi-stage looped thermoacoustic engines have attracted much attention in recent years ${ }^{[1]}$. This paper presents the looped 2-stage thermoacoustic prime movers schematically shown in Figure 1, which are composed of 2 thermoacoustic cores (including hot heat exchangers, regenerators and main cold heat exchangers) connected by resonators, thermal buffer tubes and secondary cold heat exchangers in series. The two thermoacoustic cores can be arranged in asymmetric or symmetric configuration. The total loop length is about $8 \mathrm{~m}$. Main dimensions of the thermoacoustic core are listed in Table 1. A funnel-shaped fluid director is inserted between the thermoacoustic core and resonator to reduce the flowing loss.
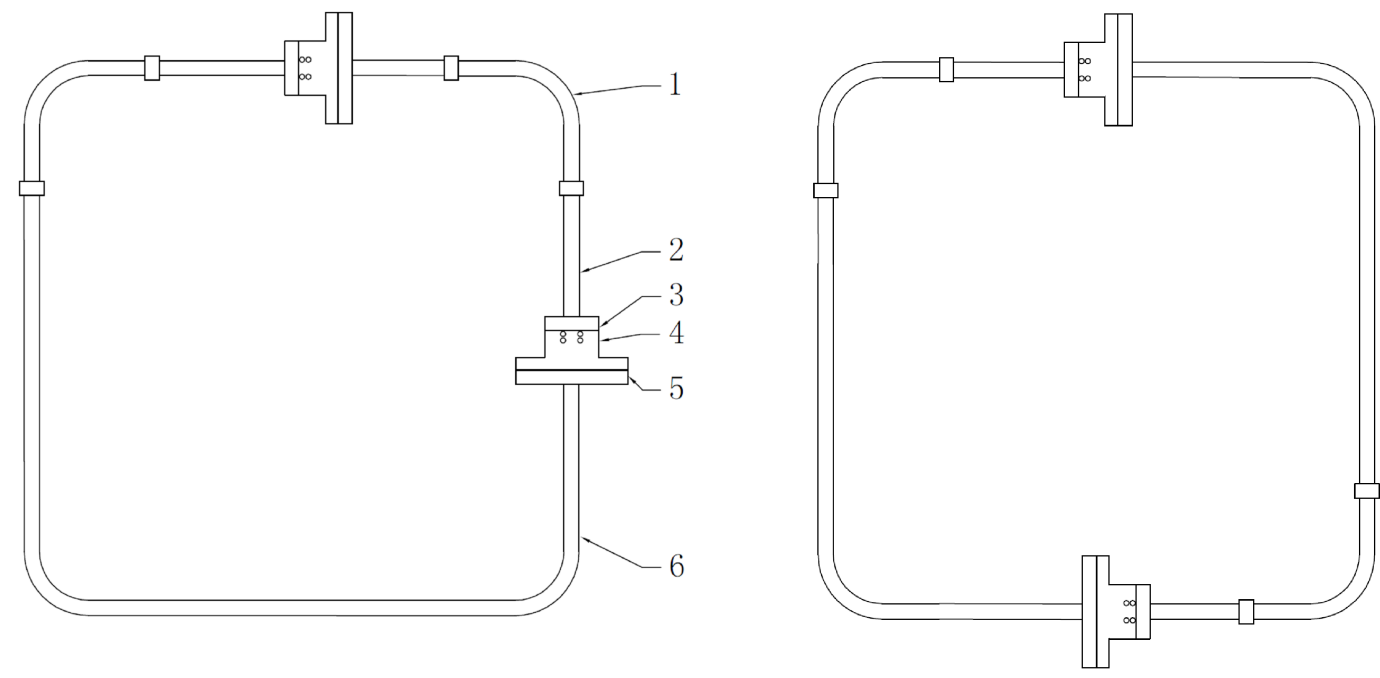

Figure 1: Schematic of the looped 2-stage thermoacoustic prime movers: 1. Secondary cold heat exchanger; 2. Thermal buffer tube; 3. Hot heat exchanger; 4. Regenerator; 5. Main cold heat exchanger; 6. Resonator.

Table 1: Dimensions of the thermoacoustic core

\begin{tabular}{lccc}
\hline Components & $\begin{array}{c}\text { Hot heat } \\
\text { exchanger }\end{array}$ & Regenerator & $\begin{array}{c}\text { Main cold heat } \\
\text { exchanger }\end{array}$ \\
\hline Length $(\mathrm{mm})$ & 30 & 30 & 30 \\
\hline Diameter $(\mathrm{mm})$ & 99 & 99 & 99 \\
\hline
\end{tabular}


Numerical simulations with the DeltaEC program ${ }^{[2]}$ and systematic experiments on the looped 2-stage thermoacoustic engine with an asymmetric configuration (see Figure 1 on the left) have been conducted with helium as the working fluid. Figure 2 presents the relation between pressure amplitude and heating temperature with the filling pressure of $2 \mathrm{MPa}$, containing the results from both experiments and simulations. Figure 3 shows the onset temperatures under different filling pressures in our experiments. A lowest onset temperature of $64^{\circ} \mathrm{C}$ (under a cooling temperature of $13^{\circ} \mathrm{C}$ ) was achieved with the filling pressure of $2.3 \mathrm{MPa}$.

A symmetric configuration of the thermoacoustic prime mover (see Figure 1 on the right), in which the two thermoacoustic cores are placed with a distance of half-wavelength, has also been tested, but failed to oscillate even at $600{ }^{\circ} \mathrm{C}$ with helium as working fluid.

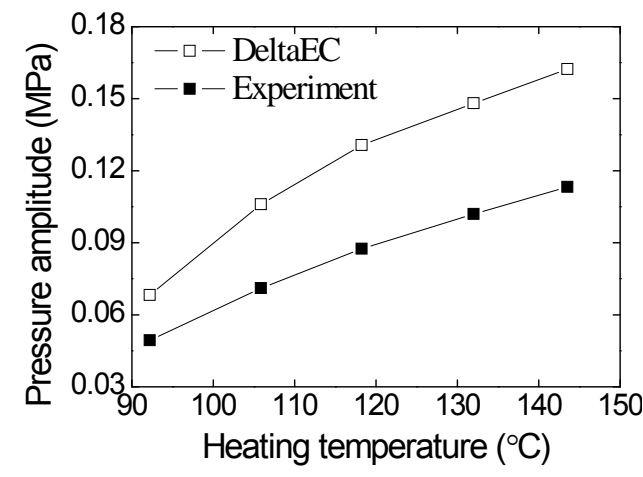

Figure 2: Pressure amplitude versus heating temperature

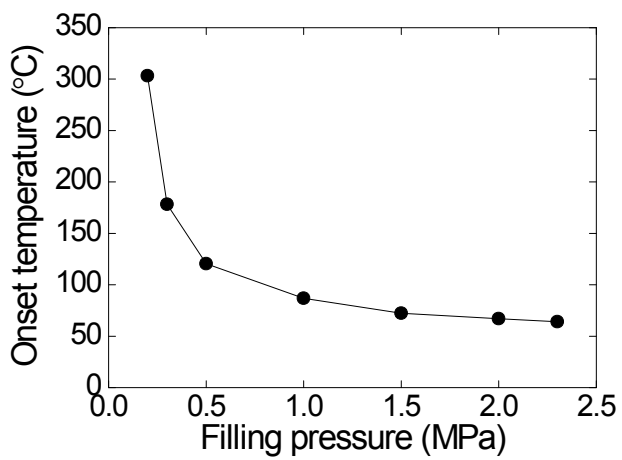

Figure 3: Onset temperature versus filling pressure

In summary, the results show that the looped 2-stage thermoacoustic prime mover with an asymmetric configuration can oscillate under a quite low heating temperature, showing the promising usability of low-grade heat source.

\section{Acknowledgements}

This work is supported by the National Natural Sciences Foundation of China (No. 51276154).

\section{References}

[1] de Blok K. Novel 4-stage Traveling Wave Thermoacoustic Power Generator, Proceedings of ASME 2010 3rd Joint US-European Fluids Engineering Summer Meeting and 8th International Conference on Nanochannels, Microchannels, and Minichannels, 2010, Montreal, Canada , 2-4 August,(2010).

[2] Ward B., Clark J., Swift G. Design Environment for Low-amplitude Thermoacoustic Energy Conversion (DeltaEC software), Version 6.2, Los Alamos National Laboratory (2008). 